\title{
Antimicrobial Stewardship Initiative in Treatment of Urinary Tract Infections at a Rehabilitation and Complex Continuing Care Hospital
}

\author{
Anne Cheung, Gulzar Karmali, Sandina Noble, and Howard Song
}

\section{INTRODUCTION}

$\mathrm{W}$

ith the trend toward increasing antimicrobial resistance and the increasing number of serious infections caused by organisms resistant to commonly used antimicrobial agents, health agencies around the world continue to focus on strategies to address and curtail these problems. ${ }^{1,2}$ In Canada, the need for antimicrobial stewardship has been recognized as a patient safety issue by the Institute for Safe Medication Practices Canada $^{3}$ and Public Health Ontario. ${ }^{4}$ Since January 2013, Accreditation Canada has included antimicrobial stewardship as a Required Organizational Practice for all health care institutions. ${ }^{5}$ However, most of the current literature and strategies related to antimicrobial stewardship apply to acute care settings. This article describes an antimicrobial stewardship initiative that was implemented at West Park Healthcare Centre (WPHC), a 270-bed rehabilitation and complex continuing care teaching hospital located in Toronto, Ontario, which specializes in the management of chronic lung diseases, diabetes mellitus, stroke, amputation, and musculoskeletal illness.

The establishment of an antimicrobial stewardship program (ASP) was approved by WPHC senior management in May 2011. Initially, the ASP team consisted of the pharmacy manager (G.K.), an ASP pharmacist (A.C., 1 day/week), and 2 infection prevention and control (IPAC) practitioners (including S.N.). In July 2011, an infectious disease physician (H.S.) was hired as a staff member (1 day/week), and this person worked as an ad hoc member of the ASP team in developing ASP guidelines for WPHC. By October 2012, a formal ASP Committee had been created as a subcommittee of the Pharmacy and Therapeutics Committee, with the vicepresident programs as its executive champion. The ASP
Committee's core membership consisted of the infectious disease physician (chair), the pharmacy manager (who subsequently became manager of both the Pharmacy and IPAC Departments), the ASP pharmacist (increased to 3 days/week), a nurse practitioner, and an IPAC practitioner. In 2014, the manager of Information Technology Services joined the ASP Committee. Representatives from other health care disciplines were included on an ad hoc basis according to the needs of various ASP initiatives.

\section{ANTIMICROBIAL USE IN URINARY TRACT INFECTIONS}

The first ASP initiative focused on use of antimicrobials for urinary tract infections (UTIs). UTIs are the most commonly reported bacterial infection in long-term care facilities, accounting for up to $50 \%$ of antibiotics used. ${ }^{6-8} \mathrm{~A}$ large proportion of these cases are thought to represent asymptomatic bacteriuria. ${ }^{8,9}$ Routine screening for asymptomatic bacteriuria is discouraged, because treatment of asymptomatic cases has not been shown to have any clinical benefit and may contribute to adverse drug reactions or re-infection with drug-resistant organisms. ${ }^{6,7}$

At the study institution, a number of problematic practices were observed, including the submission of urine samples for culture for all admissions on one inpatient unit, irrespective of symptoms or signs, as well as the culturing of urine samples on the basis of appearance (e.g., cloudiness) and smell. At WPHC, registered nurses have the authority to send urine specimens for culture and sensitivity $(\mathrm{C} \& S)$ testing under a medical directive. Of equal concern was the decreasing rate of ciprofloxacin sensitivity in urine isolates of Escherichia coli, which fell below $60 \%$ in 2011 . With the aforementioned in mind, the following 
goals were established: to develop an evidence-based guideline for use of antimicrobials in UTIs (the UTI guideline) and to provide guidance on collection of urine specimens for C\&S testing.

Development of the UTI guideline (Table 1) was based on guidelines from the Infectious Diseases Society of America ${ }^{10,11}$ and review of WPHC's 2010 and 2011 antibiograms. According to these antibiograms, the predominant uropathogens at WPHC were E. coli (over $50 \%$ of isolates) and Enterococcus spp. (about 30\%). Therefore, empiric antibiotic regimens at the study institution needed to cover both of these uropathogens. Neither ciprofloxacin nor trimethoprim-sulfamethoxazole (TMP/SMX) could be recommended for empiric therapy because the sensitivity of $E$. coli to these agents was below $60 \%$ and $80 \%,{ }^{10}$ respectively. Furthermore, neither ciprofloxacin nor TMP/SMX is considered effective against Enterococcus spp.

Nitrofurantoin was therefore recommended as first-line therapy for uncomplicated cystitis, provided the patient's creatinine clearance was above $50 \mathrm{~mL} / \mathrm{min} .{ }^{12}$ Amoxicillinclavulanic acid was recommended as second-line therapy for uncomplicated cystitis and as first-line therapy for mild to moderate pyelonephritis or complicated UTI. Ciprofloxacin could be used as third-line empiric treatment for uncomplicated cystitis and second-line empiric treatment for mild to moderate pyelonephritis or complicated UTI. Patients with more severe complicated UTI or pyelonephritis, characterized by hemodynamic instability, high fever, or inability to tolerate oral antibiotics, were to be treated with IV antibiotics. The UTI guideline recommended that the initial antibiotic choice be reviewed and modified as appropriate, according to reported susceptibilities. If no urine C\&S data were available (because no specimen had been sent or because the laboratory either did not receive the specimen or rejected it because of possible contamination) and the patient's condition improved on empiric treatment, the UTI guideline recommended completing the course of treatment. Guidance on the duration of treatment, according to type of UTI, sex, and the selected antibiotic (either the empiric choice or based on available C\&S results) was also included.
In addition to providing recommendations on antimicrobial use, the UTI guideline included an algorithm of the clinical indications for collection of urine for C\&S testing in patients with and without an indwelling catheter (Figure 1). ${ }^{13}$ Because the microbiology laboratory analysis and report depended on the method of urine collection, the UTI guideline included microbiologic criteria for a positive culture result from a midstream urine sample versus a catheter specimen, as well as providing clear labelling instructions defining the method of urine collection. Specifically, when 3 or more organisms were identified in a midstream specimen, the specimen would typically be rejected by the laboratory because of possible contamination; in contrast, up to 3 dominant organisms would be characterized from a catheter specimen.

The UTI guideline was approved by the Pharmacy and Therapeutics Committee and the Medical Advisory Committee and was implemented hospital-wide by the end of 2011. To reinforce awareness and use of the UTI guideline, the following measures were implemented:

- Education for physicians: Review of the UTI guideline was conducted by the ASP pharmacist on a 1:1 basis with the most responsible physician for each rehabilitation and complex continuing care unit at a prearranged 20-min session (December 2011).

- Education for clinical pharmacists: Starting in July 2012, group teaching sessions were conducted by the ASP pharmacist and infectious disease physician on the UTI guideline and empiric regimens, including review of UTI case studies to reinforce application of the guideline.

- IPAC-led education for nurses: In 2013, education on appropriate indications for urine collection and on the UTI guideline was implemented using a decentralized approach, whereby an IPAC practitioner or advanced practice nurse conducted the education sessions on the rehabilitation and complex continuing care units, to facilitate attendance by nurses. This decentralized education was repeated over a 3-month period (July to September 2013) in a more intense nursing education

Table 1. Summary of Guideline for Treatment of Urinary Tract Infection (UTI) at West Park Healthcare Centre*

\begin{tabular}{|c|c|c|}
\hline Type of UTI & First-Line Empiric Therapy & Alternative Empiric Therapy \\
\hline Uncomplicated cystitis & $\begin{array}{l}\text { Nitrofurantoint } \\
\text { monohydrate/macrocrystals } \\
100 \text { mg PO q12h }\end{array}$ & $\begin{array}{l}\text { Amoxicillin-clavulanic acid } \neq \\
500 \mathrm{mg} / 125 \mathrm{mg} \text { PO q8h }\end{array}$ \\
\hline $\begin{array}{l}\text { Mild to moderate pyelonephritis or } \\
\text { complicated UTI (hemodynamically stable, } \\
\text { no vomiting) }\end{array}$ & $\begin{array}{l}\text { Amoxicillin-clavulanic acid } \\
875 \mathrm{mg} / 125 \mathrm{mg} \text { PO q12h }\end{array}$ & Ciprofloxacin 500 mg PO q12h \\
\hline $\begin{array}{l}\text { Severe pyelonephritis or complicated UTI } \\
\text { (high fever, sepsis, vomiting) }\end{array}$ & $\begin{array}{l}\text { Ampicillin } 1 \mathrm{~g} \text { IV q6h + } \\
\text { gentamicin } 3 \mathrm{mg} / \mathrm{kg} \mathrm{IV} \mathrm{q24h}\end{array}$ & Ceftriaxone 1g IV q24h \\
\hline
\end{tabular}




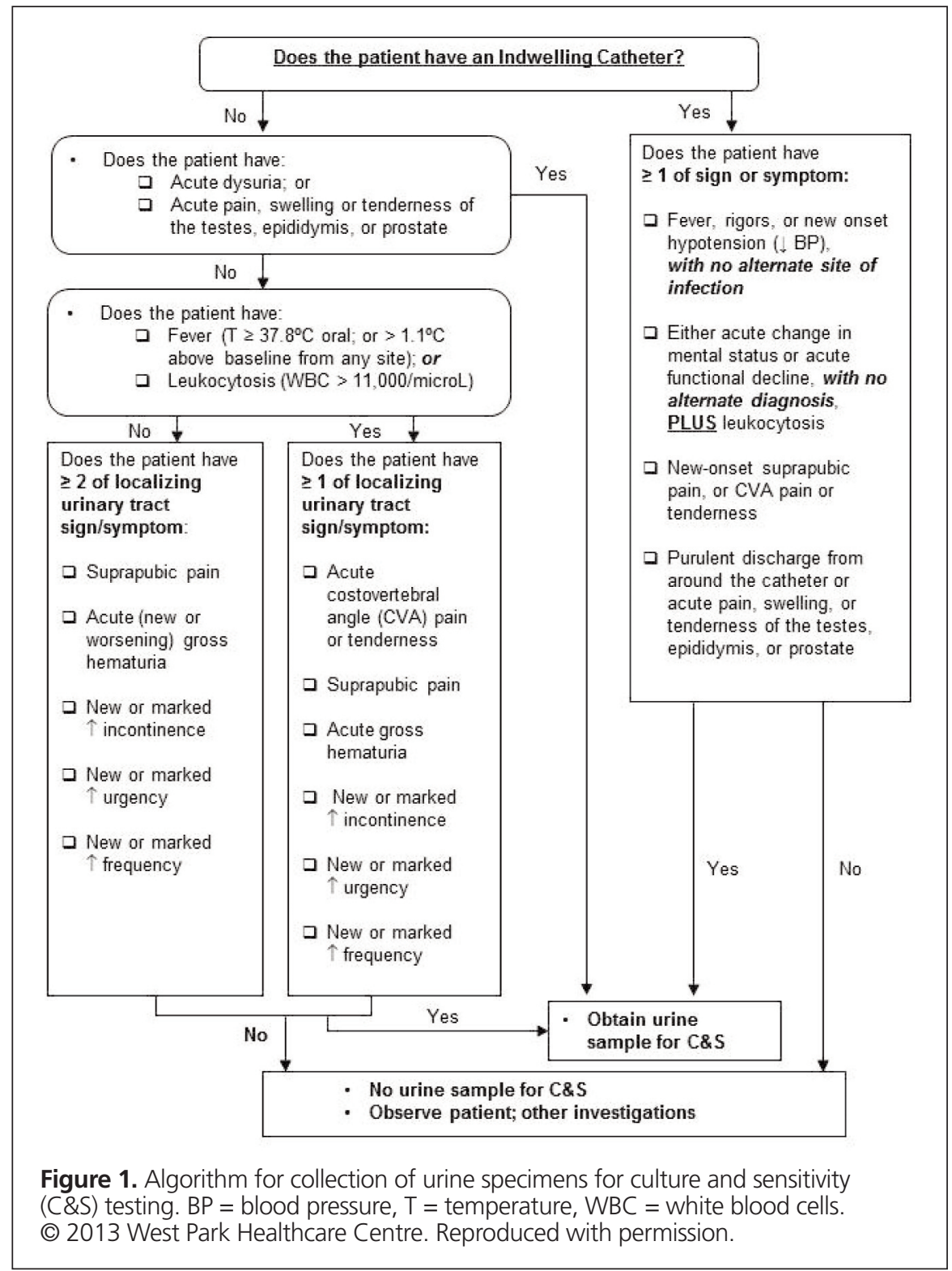

campaign, with the support of the unit managers, senior management, and human resources in developing a schedule and mandating attendance to at least one session. Following each 2013 education session, the educator used a short questionnaire (Appendix 1) to reinforce with participants the key learnings about the UTI guideline and the urine collection algorithm. Of 256 active nurses, 247 participated in these education sessions, for an overall participation rate of $96.5 \%$ by the end of the nursing education campaign.

- Electronic access to the UTI guideline for all staff through the hospital intranet (January 2013).

- Modification of order entry for urine specimens in the computerized physician order entry (CPOE) system to include the type of urine specimen (e.g., midstream urine, in-and-out catheter) (May 2013).

- Development of electronic UTI order sets in the CPOE system (April 2014).

- Development of a pocket card summarizing the empiric antibiotic regimens (one side) and the urine collection algorithm (reverse). Pocket cards were distributed to physicians and nurse practitioners by the infectious disease physician during a professional staff association meeting and to clinical pharmacists and IPAC practitioners (September and October 2014).

\section{CLINICAL IMPACT}

Since implementation of the UTI guideline, annual antibiograms have continued to support use of nitrofurantoin and amoxicillin-clavulanic acid as first- and second-line empiric 

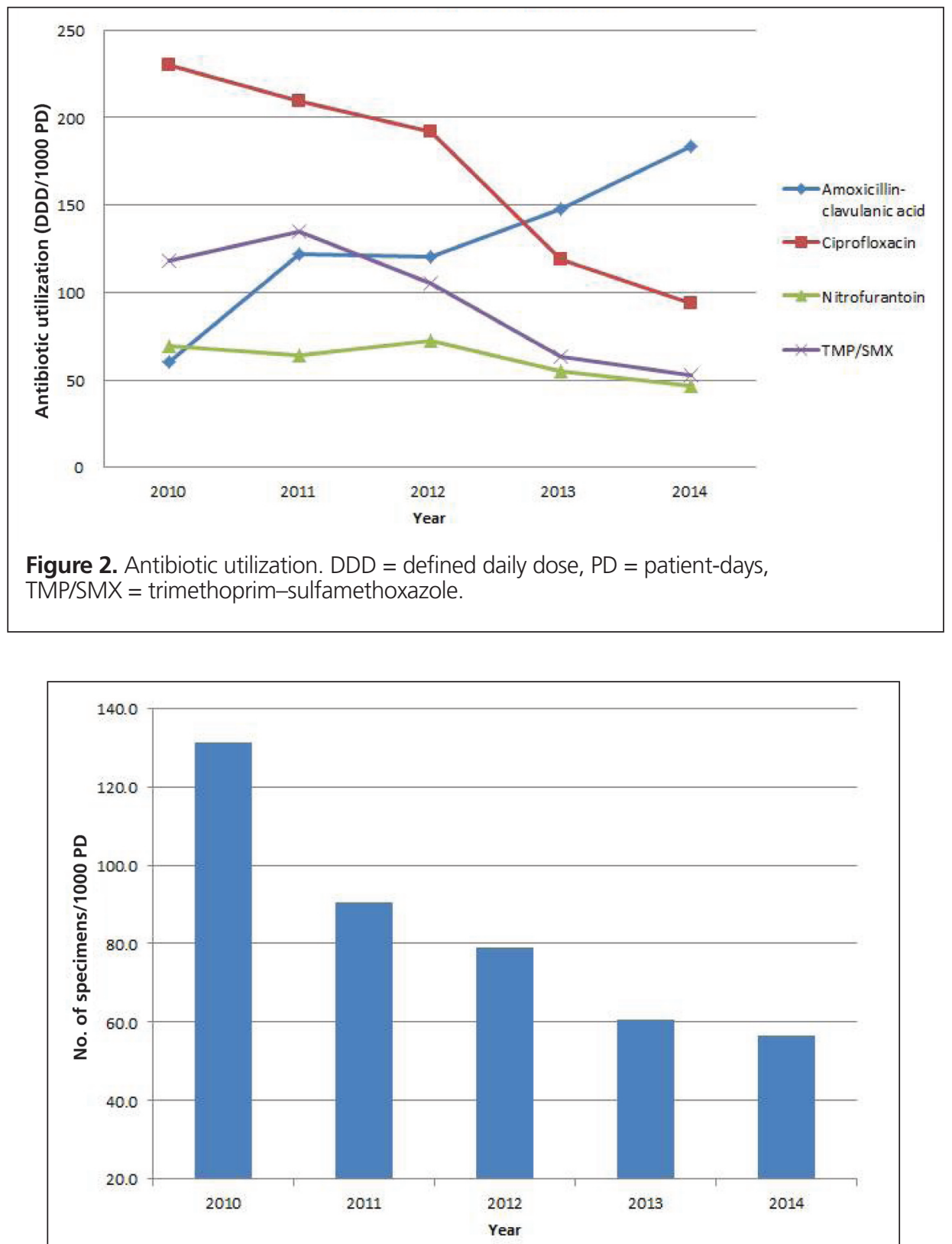

Figure 3. Collection of urine specimens for culture and sensitivity testing. $\mathrm{PD}=$ patient-days.

therapy against the 2 most common urinary pathogens at WPHC, E. coli and Enterococcus spp.

Aggregate drug utilization data, represented by defined daily dose per 1000 patient days (DDD/1000 PD), were calculated for amoxicillin-clavulanic acid, ciprofloxacin, nitrofurantoin, and TMP/SMX for all rehabilitation and complex continuing care units, except for the tuberculosis unit (the rationale for this exclusion was that amoxicillin-clavulanic acid is used extensively on the tuberculosis unit for the indication of tuberculosis, and the incidence of UTI is very low). The aggregate data showed that use of amoxicillin-clavulanic acid surpassed use of ciprofloxacin, while use of ciprofloxacin and TMP/SMX decreased, and use of nitrofurantoin remained unchanged (Figure 2). Although the antibiotic utilization trends appear consistent with the UTI guideline, the $\mathrm{DDD} / 1000 \mathrm{PD}$ data are aggregated and not specific for the treatment of UTIs; as such, the observed changes in utilization cannot be attributed solely to the UTI guideline.

Following the IPAC-led education for nurses on the urine collection algorithm, the total number of urine specimens sent for culture per 1000 PD decreased by $40.0 \%$ in 2012 relative to 2010 (Figure 3). This decrease was achieved primarily by 


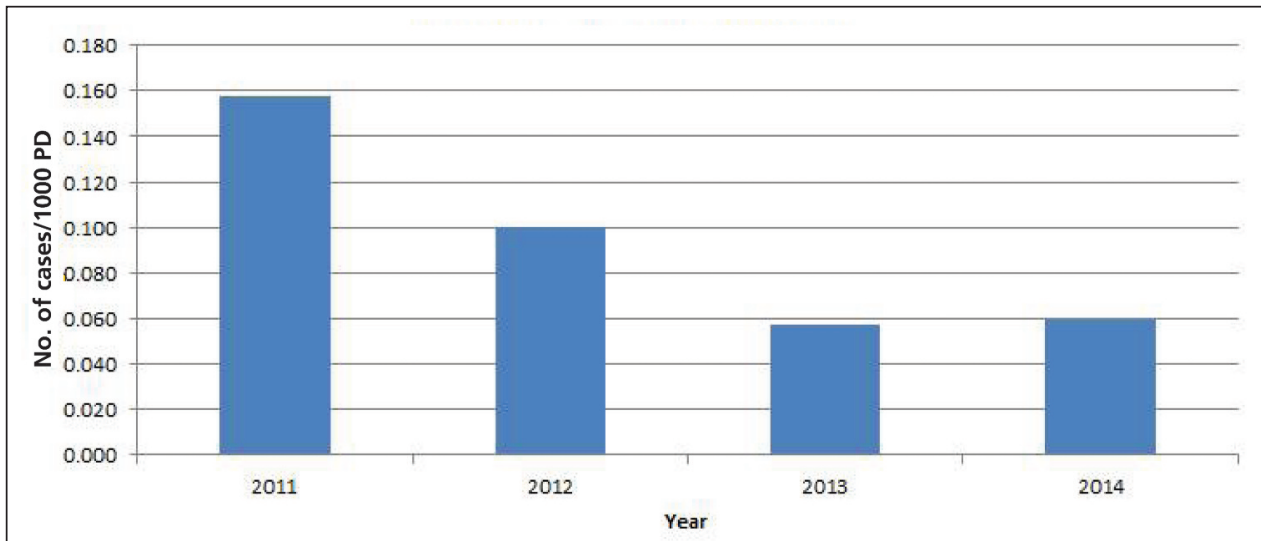

Figure 4. Rate of health care-associated Clostridium difficile infections. PD = patient-days.

stopping a problematic practice on one inpatient unit whereby urine specimens were routinely sent for C\&S testing upon admission. A further $22.9 \%$ decrease in 2013 relative to 2012 was observed after the intensive targeted education campaign for nurses over the period July to September 2013 (Figure 3).

There was a concern that the increased use of amoxicillinclavulanic acid, a broad-based antibiotic, might encourage the development of Clostridium difficile infections. If an increase in the institutional rate of health care-associated $C$. difficile infection were observed, further investigation would be needed to assess whether the UTI guideline had contributed to the increase. However, a review of rates of health care-associated C. difficile infection from 2011 to 2014 showed a downward trend (Figure 4). Again, this trend could not be attributed solely to the UTI guideline, as there were other institutional initiatives (e.g., IPAC-led education on hand hygiene) that could have contributed to this favourable outcome.

\section{CONCLUSION}

This article has described the implementation of an ASP initiative in a rehabilitation and complex continuing care hospital, which specifically targeted the use of antibiotics in the management of UTIs. The core strategy was to develop an institutional UTI guideline, which emphasized evidence-based antibiotic treatment and reinforced the collection of urine only in symptomatic cases. Key success criteria included the involvement of an infectious disease physician in development and review of the UTI guideline and a targeted education campaign for nurses, led by an IPAC practitioner, concerning the urine collection algorithm. In summary, the uptake of these guidelines relied heavily on a hospital-wide collaborative effort by a number of interprofessional practices, including IPAC, pharmacy, nursing, and information technology.

\section{References}

1. Global action plan on antimicrobial resistance. Geneva (Switzerland): World Health Organization; 2015 [cited 2014 Dec1]. Available from: www.who.int/ drugresistance/global_action_plan/en/

2. Antibiotic/antimicrobial resistance. Atlanta (GA): Centers for Disease Control and Prevention; 2015 [cited 2014 Dec 1]. Available from: www. cdc.gov/drugresistance/

3. Antimicrobial stewardship. ISMP Can Saf Bull. 2010;10(4):1-2.

4. Antimicrobial stewardship. Toronto (ON): Public Health Ontario; [cited 2014 Dec 1]. Available from: https:/www.publichealthontario.ca/en/ BrowseByTopic/InfectiousDiseases/AntimicrobialStewardshipProgram/Pages /Antimicrobial-Stewardship-Program.aspx

5. Antimicrobial stewardship. In: Required organizational practices handbook 2017. Ottawa (ON): Accreditation Canada; 2016. pp. 40-1.

6. Nicolle LE. Urinary tract infection in long-term care facility residents. Clin Infect Dis. 2000;31(3):757-61.

7. Genao L, Buhr GT. Urinary tract infections in older adults residing in long-term care facilities. Ann Longterm Care. 2012;20(4):33-8.

8. Genitourinary infections. In: Anti-infective guidelines for communityacquired infections. Toronto (ON): Partners for Appropriate Anti-infective Community Therapy; 2013. pp. 68-71.

9. Nicolle LE, Bradley S, Colgan R, Rice JC, Schaeffer A, Hooton TM; Infectious Diseases Society of America; American Society of Nephrology; American Geriatric Society. Infectious Diseases Society of America guidelines for the diagnosis and treatment of asymptomatic bacteriuria in adults. Clin Infect Dis. 2005;40(5):643-54.

10. Gupta K, Hooton TM, Naber KG, Wullt B, Colgan R, Miller LG, et al. International clinical practice guidelines for the treatment of acute uncomplicated cystitis and pyelonephritis in women: a 2010 update by the Infectious Diseases Society of America and the European Society for Microbiology and Infectious Diseases. Clin Infect Dis. 2011;52(5):e103-20.

11. Hooton TM, Bradley SF, Cardenas DD, Colgan R, Geerlings SE, Rice JC, et al. Diagnosis, prevention, and treatment of catheter-associated urinary tract infection in adults: 2009 international clinical practice guidelines from the Infectious Diseases Society of America. Clin Infect Dis. 2010;50(5):625-63.

12. Gilbert DN, Moellering RC, Eliopoulos GM, Chambers HF, Saag MS, editors. The Sanford guide to antimicrobial therapy. 43rd ed. Sperryville (VA): Antimicrobial Therapy, Inc; 2013. p. 207.

13. Stone ND, Ashraf MS, Calder J, Crnich CJ, Crossley K, Drinka PJ, et al.; Society for Healthcare Epidemiology Long-Term Care Special Interest Group. Surveillance definitions of infections in long-term care facilities: revisiting the McGeer criteria. Infect Control Hosp Epidemiol. 2012; 33(10):965-77. 
Anne Cheung, BScPhm, MBA, is with West Park Healthcare Centre, Toronto, Ontario.

Gulzar Karmali, BScPhm, is with West Park Healthcare Centre, Toronto, Ontario.

Sandina Noble, BSC, RN, CIC, is with West Park Healthcare Centre, Toronto, Ontario.

Howard Song, MD, PhD, FRCPC, is with West Park Healthcare Centre, Toronto, Ontario.

Competing interests: None declared.

\section{Address correspondence to:}

Anne Cheung

West Park Healthcare Centre

82 Buttonwood Avenue

Toronto ON M6M 2J5

e-mail: Anne.Cheung@westpark.org

Funding: None received.

Acknowledgement: The authors thank Sandra A N Walker, BSC, BScPhm, PharmD, FCSHP, Department of Pharmacy, Sunnybrook Health Sciences Centre, for her guidance on the manuscript.

\section{Appendix 1. Questionnaire for nurses following education sessions about urinary tract infection (UTI)}

1. Patient " $\mathrm{A}$ " has a positive urine culture but no other clinical symptoms. She needs an antibiotic to be started.

True or False

2. Patient " $\mathrm{B}$ " is a new admission. You need to collect a urine sample for C\&S as part of an admission screen.

True or False

3. Patient "C" has an indwelling catheter and has developed a fever. A urine sample should be collected.

True or False

4. An RPN is worried about a patient. Patient " $D$ " does not have an indwelling catheter but has developed acute hematuria and marked increase in frequency.

A) Can the RPN send a urine sample for C\&S?

B) Should a urine sample be collected for C\&S?

5. Patient " $\mathrm{D}$ " has strong-smelling urine, which is dark in colour. He has no other signs and symptoms of a UTI. He needs a specimen collected for C\&S.

True or False
6. Patient "E" does not have an indwelling catheter but has developed a marked increase in incontinency and urgency. A urine specimen should be collected for C\&S.

True or False

7. Patient "F", an elderly patient without an indwelling catheter, has a change in mental status. Should you send a urine specimen for $\mathrm{C} \& S$ ?

Yes or No

8. Patient " $G$ " with an indwelling catheter has developed a fever and new onset of suprapubic pain, and has purulent discharge from around the catheter. A urine specimen needs to be collected for C\&S. Should the catheter be changed before collecting the specimen?

Yes or No

$\mathrm{C} \& S$ = culture and sensitivity, $\mathrm{RPN}$ = registered practice nurse . 